\title{
Common Peroneal Nerve Palsy Secondary to a Proximal Tibiofibular Joint "Ganglion Cyst"-A Case Report and Review of Literature*
}

\author{
Mohit M. Kukreja'#, Vidyadhar G. Telang² \\ ${ }^{1}$ Tan Tock Seng Hospital, Singapore City, Singapore \\ ${ }^{2}$ Dhanvantari Hospital \& Research Centre, Mumbai, India \\ Email: "drmohitkukreja@gmail.com
}

Received 20 October 2015; accepted 17 November 2015; published 20 November 2015

Copyright (C) 2015 by authors and Scientific Research Publishing Inc.

This work is licensed under the Creative Commons Attribution International License (CC BY). http://creativecommons.org/licenses/by/4.0/

\section{(c) (i) Open Access}

\section{Abstract}

Although ganglion cysts occur frequently, their presence in the lower extremities is rare and they seldom cause peripheral nerve compression. There are enumerable case reports of intraneural ganglion involvement with the common peroneal nerve and its branches, the sural nerve, and the posterior tibial nerve but extraneural ganglion sciatic and common peroneal nerve palsy cases are quite rare. Our case, a 26 years old female patient presented with right leg tingling and radiating pain followed up gradually with progressive right sided foot drop. MRI diagnosed the lesion as a ganglion cyst and the EMG/NCV confirmed the level of compression at the right fibular head. The patient was electively operated with standard lateral approach and the ganglion cyst engulfing the common peroneal nerve was excised. The cyst was traced to the base of its articular origin, excised and stalk ligated to prevent recurrence. At 1 year, there were no signs of recurrence and the patient was symptomatically free with no residual weakness. We, thus, report to you a rare cause of common peroneal nerve palsy - a proximal tibio-fibular joint "ganglion cyst"; a cause of foot drop which can be completely reversible if treated appropriately, its implications and thus, making its early diagnosis significant.

\section{Keywords}

Ganglion, Tibiofibular, Peroneal, Excision

\footnotetext{
*The authors have obtained the patient's guardians informed written consent for print and electronic publication of the case report. ${ }^{*}$ Corresponding author.
} 


\section{Introduction}

Although ganglion cysts occur frequently, their presence in the lower extremities is rare and they seldom cause peripheral nerve compression. The most common nerve involved in the upper extremity is the ulnar nerve while passing through the cubital tunnel [1]. English literature has produced previous case reports of intraneural ganglion involvement with the common peroneal nerve and its branches, the sural nerve, and the posterior tibial nerve but extraneural ganglion sciatic and common peroneal nerve palsy cases are seldom documented [2]. There have been reports of intraneural ganglion involvement with CPN branches [3] and hypothesis that the peroneal ganglia arise from the proximal tibiofibular joint [4] but historically, the first reported case of compression peroneal nerve neuropathy by a ganglion cyst was produced by Sultan in 1921 [7]. One perceivable reason for less documentation of such cases may be that an orthopaedic surgeon is rarely referred directly for a clinically pure neuropathic foot drop.

The common peroneal nerve is derived from the dorsal branches of L4, L5, S1 and S2 roots [5]. After descending along the lateral side of the popliteal fossa, the common peroneal nerve (CPN) winds round the head of the fibula and divides into the superficial peroneal nerve (SPN) and the deep peroneal nerve (DPN) [5]. The SPN innervates the peroneus longus and peroneus brevis muscles. The DPN supplies the muscles of the anterior compartment of the leg [5]. Together these muscles are responsible for the dorsiflexion of the ankle and extension of the toes. It also innervates the intrinsic muscles of the foot [6].

We, thus, report to you a rare cause of common peroneal nerve palsy—a proximal tibio-fibular joint "ganglion cyst"; a cause of foot drop which can be completely reversible if treated appropriately, its implications and thus, making its early diagnosis significant.

\section{Presentation of the Case}

Our case, a 26 years old female, presented with right sided knee discomfort and radiating pain in the calf and leg area since last 6 months. The discomfort was localized to the lateral aspect of the knee, was nagging in nature and more symptomatic on prolonged standing. The pain was radiating to the right lateral aspect of the leg and foot area and burning in nature. This was followed by weakness in the right foot, inability to dorsiflex and walking with a slap-hammer gait. The patient also complained of $1^{\text {st }}$ great toe weakness and numbness. There was no history of trauma to the knee. Also, there was lack of any systemic illness or lower back complaints. There was no significant past medical or surgical history.

On clinical examination, the patient walked with a high-steppage gait with inability to dorsiflex the right foot. There was no visible swelling in the knee or the leg. Neurological weakness was documented with $\mathrm{DF}=2 / 5$ in the right foot (MRC Grading) with 20\% sensation in the $1^{\text {st }}$ web space and the lateral aspect of the right foot. Positive Tinel's sign was localized to the right fibular head. Spine examination and peripheral pulses were normal.

Figure 1 shows the clinical photographs of both lower limbs of this patient showing right sided foot drop with inability to dorsiflex the right foot actively. The power in the toe flexors $=2 / 5$.

Figure 2 shows the Bilateral knee X-rays of the patient both AP and Lateral views which did not show any significant abnormality.

We followed up the patient with MRI of the Right knee.

Figure 3 shows the MRI images of the Right knee with the Coronal, Sagittal and Axial cuts of the T2W images. The cyst was localize with its exact dimensions and its relation to the Right tibio-fibular joint. It was reported as Right sided ganglion cyst at the fibular head level with T2 W hyperintense and T1W hypointense collection and minimal synovial effusion in the proximal tibiofibular joint. The cyst was reported as lobular and multiloculated approximately $2 \mathrm{~cm} \times 1.5 \mathrm{~cm} \times 1 \mathrm{~cm}$.

We also did the EMG/NCV to know more about the level of the lesion and confirm ganglion cyst at the proximal tibio-fibular syndesmosis as a cause of the foot drop. It was reported as complete popliteal nerve motor conduction block at the fibular head with absent sensory potentials in the superficial peroneal nerve. No abnormalities were found in the muscles innervated by the tibial nerve. Thus, a complete degenerative right lateral popliteal neuropathy at the fibular head was diagnosed.

After further preoperative work up, the patient was taken for a synovial cyst excision without much delay.

We took the standard lateral approach. The incision was a curvilinear lateral incision spanning the fibular head region and extending posteriorly. 


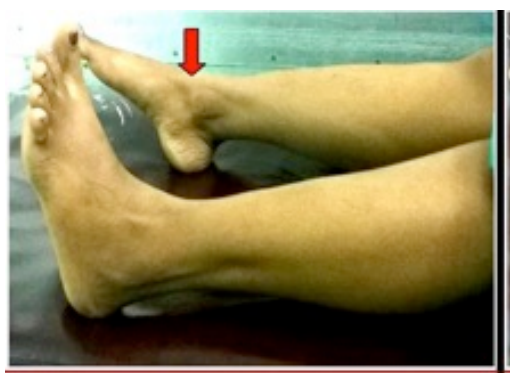

(a)

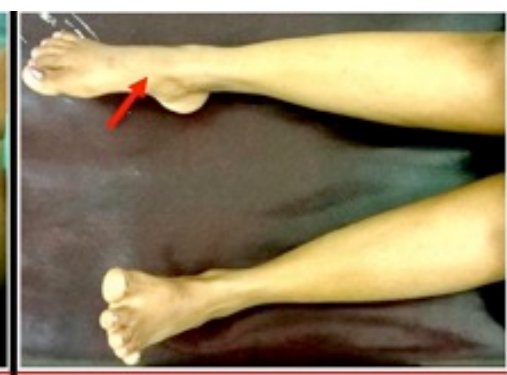

(b)

Figure 1. Clinical photographs [(a) and (b)] of the patient showing right sided foot drop with inability to dorsiflex the right foot actively. The thick vertical arrow in (a) and the thin oblique arrow in (b) points to the foot drop. There was Grade 2/5 muscle strength of the toe flexors.

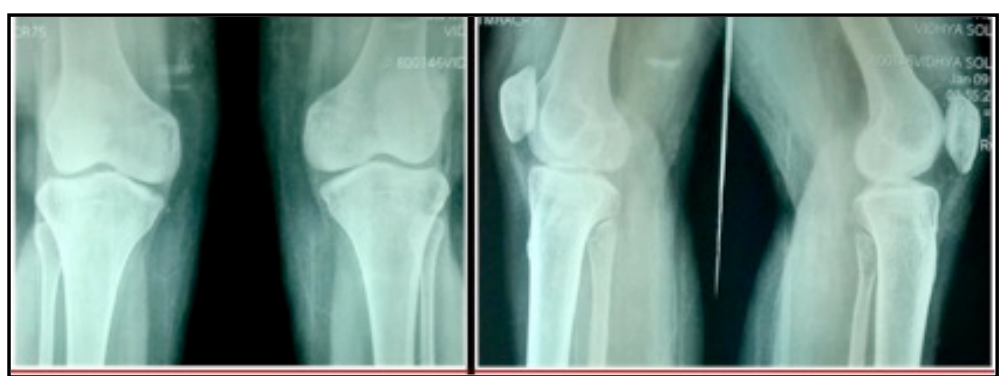

(a)

(b)

Figure 2. X-ray of bilateral knees in symmetrical position. (a) shows the anteroposterior view of both knees and (b) shows the lateral views. There were no abnormal findings in the X-rays.


Figure 3. MRI images of the right knee with the coronal (a); axial (b) and sagittal (c) cuts of the T2W images. The red circle in (a); the oblique thick arrow in (b) and the oblique thin arrow in (c) point to the hyperintense globular fluid-filled cystic lesion.

Figure 4 has the left photograph showing the curvilinear nature of the incision and the right photograph showing the bulge in the deep fascia because of the underlying cyst.

The deep fascia was incised in line of the skin incision and the CPN isolated as it hooks the fibular neck. The ganglion cyst extended out of the proximal tibio-fibular joint region in a multilocular fashion adhering to the common peroneal nerve before its anatomical bifurcation. 
Figure 5 depicts the multilocular nature of the cyst, its proximity to the tibio-fibular joint and its adherence to the Common peroneal nerve before its bifurcation.

The nerve was isolated by meticulous dissection from the cyst and the soft tissue. The contour of the cyst was such that it was proximal to the bifurcation of the CPN into its branches. Thus the compression was directly on the CPN rather than its branches thus affecting the lateral and the anterior compartment innervation.

Figure 6 exhibits the exact anatomy of the cyst and its relation to the respective branches of the CPN. The compression of the CPN is direct and not on its branches.



Figure 4. The left photograph (a) shows the curvilinear nature of the incision and the right photograph (b) shows the bulge in the deep fascia because of the underlying cyst seen immediately after the incision.

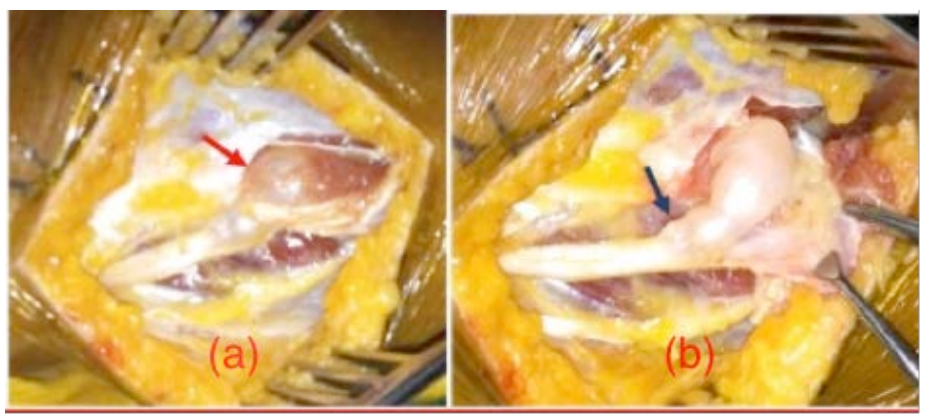

Figure 5. Multilocular nature of the cyst, its proximity to the tibio-fibular joint and its adherence to the common peroneal nerve before its bifurcation. (a) (red arrow) shows its nerve proximity and (b) (black arrow) shows its multilocularity and its nerve adherence.

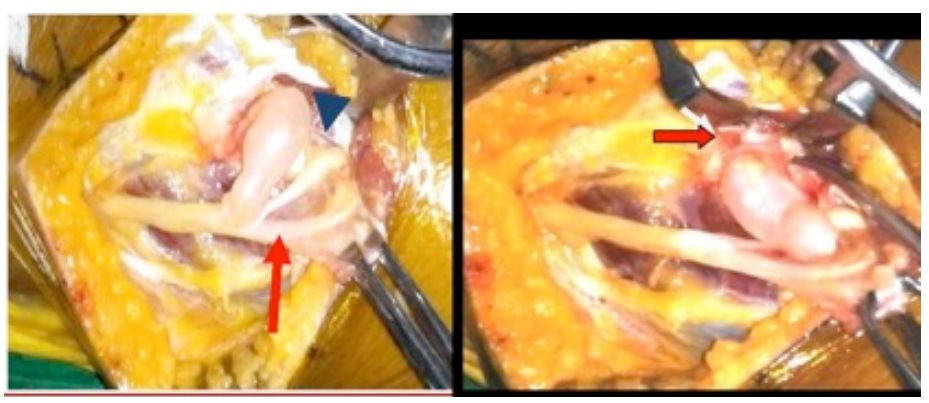

Figure 6. The cyst with its anatomical relations with the proximal tibio-fibular joint and the respective branches of the CPN (vertical red arrow). The compression of the CPN is direct and not on its branches. The black arrow head and the horizontal red-black arrow points to the articular stalk of the cyst. 
The cyst was traced to the depth of its articular stalk from the proximal tibio-fibular joint. The articular branch of the cyst was ligated and excised. The excised cyst, on histopathological tissue examination, came out to be a fibrous outermost covering with synovial lining.

Figure 7 shows the final intraoperative photograph after complete cyst excision and Figure 8 shows the histopathological confirmation of the cystic nature of the lesion.

The patient was discharged on the 3 rd postoperative day after the first check-dress, the suture removal being done after 2 weeks and the patient followed up later at 4 weeks, 6 weeks, 3 monthly intervals.

3 months postoperatively, the foot drop recovered to improve motor weakness to power $=4 / 5$ with complete recovery by 6 months. Currently, at 1 year after cyst excision, there is no sign of recurrence and patient is symptomatically pain free with no residual weakness.

\section{Discussion}

Peripheral neuropathy because of ganglionic cysts are infrequent findings [2]. Since the first description of a peroneal nerve neuropathy by Sultan in 1921 [7], very few cases of the lower limb compression neuropathies have been described in the surgical literature [2]-[11]. Most of these cases have been described in adults but there have even been reports of such lesions compressing the CPN at the proximal tibio-fibular joint in as young as 12 years of age by Frank et al. [6]. The etiology may vary and the various implications are degenerative, post-traumatic, inflammatory, and euplastic processes. Clinically, the presentation may range from being completely asymptomatic to some presenting with just nonspecific pain and tenderness in the region of the cyst [6].

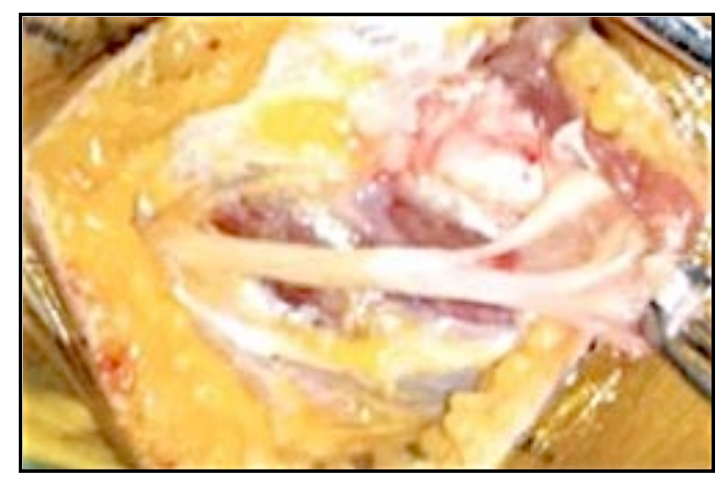

Figure 7. Intraoperative photograph after complete cyst excision.

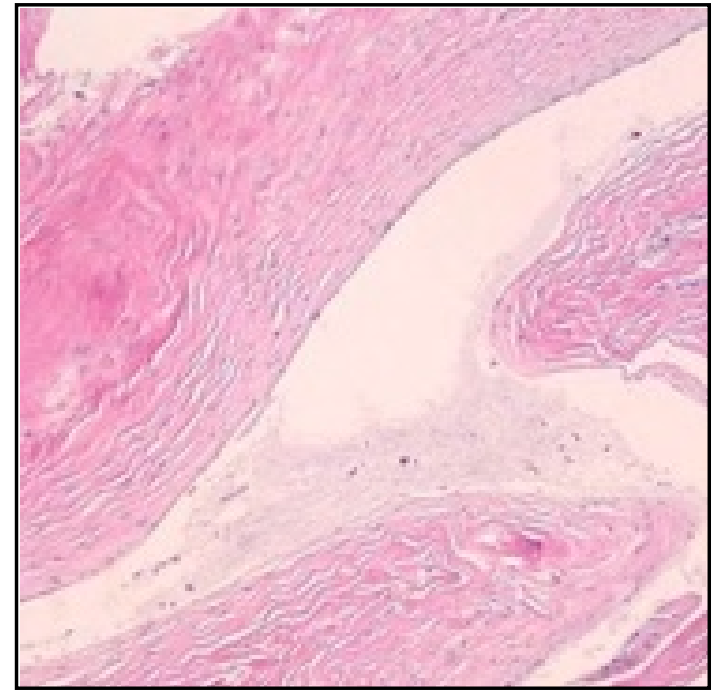

Figure 8. Shows the light (optical) microscopy histopathological slide confirming the diagnosis of a Ganglion cyst using the Hematoxylin \& Eosin stain (H \& E stain). 
The CPN is derived from L4, L5, S1, and S2 as a main division of the sciatic nerve and arises near the upper level of the popliteal fossa [2] [7] [8]. The nerve becomes most vulnerable at its entrance to the fibular tunnel, where it courses superficial to the lateral surface of the fibula just distal to the fibular head [7] [8] [12]. This superficial location gives the nerve little soft tissue protection. Mont et al. [13], in a review of 31 patients receiving operative decompression for a peroneal nerve palsy, describe bands of fibrous tissue constricting the peroneal nerve at this level which could further increase the nerve's susceptibility to compression.

Rawal et al. [4] hypothesized that the origin of peroneal nerve ganglia is the proximal tibiofibular joint, via the articular branch. Synovial cysts are mucinous filled cysts that can be found adjacent to joint capsule or tendon sheath. They can originate within perineural tissue or in an adjacent joint space or bursa and extend toward the nerve [14]. They consist of an outer fibrous coat and an inner synovial lining and contain a clear, colorless, gelatinous fluid [14].

A review of the literature suggests etiologies for peroneal nerve palsy as either intraneural (schwannoma, nerve herniation through fascia and giant plexiform neurofibromatosis) [8] [11] [15] [16] and extraneural (varicose veins, post-pneumatic compression, valgus knee total arthroplasty or proximal tibial osteotomy and ganglion cysts) [2] [4] [8] [15] [17]. Although rarely documented, intraneural ganglions located within the substance of nerves may cause direct nerve compression. This direct compression is usually acute with sudden-onset foot drop [5]. Tehli described an intraneural cyst ascending along the sciatic nerve [5]. As already mentioned, there have been many previous reports of intraneural ganglion involvement with the CPN and its branches [3]; extraneural ganglion cyst involving the CPN is rarely reported. In this case, we excluded a possible lumbar disc herniation by a lumbosacral MRI investigation although patient had an asymptomatic back. Some of the palsies can be caused by ganglia with no palpable mass which makes the diagnosis difficult [18].

Ward et al. [19] described a case of a proximal tibiofibular joint ganglion that resulted in compartment syndrome of the anterior compartment of the leg. Gibbon et al. [20] reported a case of peroneal nerve compression by a ganglion cyst in a patient who had undergone total knee arthroplasty. Cysts arising from the lateral meniscus with peroneal nerve involvement also have been described [21].

Ganglion cysts are commonly accompanied by signs of nerve irritation such as numbness, tingling, and radiating pain along the path of the compressed nerve [8]. In peroneal nerve palsies, the patients often complain of altered ambulation secondary to paretic foot dorsiflexors [8]. A high-steppage gait pattern is common due to the weakness in the tibialis anterior, the extensor hallucis longus, the extensor digitorum longus, and the peroneus longus and brevis muscles. The extensor digitorum brevis is the most profoundly affected [8]. While pain is not universal, when present, it is often related to the specific site of the CPN compression or its branches (superficial or deep) [8]. Sensory testing often shows a loss in the cutaneous distribution of the CPN and Tinel's sign is generally positive in the sensory territory of the peroneal nerve [8].

The differential diagnosis should include L5 nerve root lesion, a nerve pathology near the tendinous arch of the peroneal longus muscle, a neural sheath tumor and exostosis at the proximal lower leg [6] [9] [11] [18] [22].

Rapid diagnosis and prompt treatment of a suspicious case after confirmation tests is important; several reports emphasize the importance of early diagnosis which has implications on complete recovery of the paresis [13] [23] [24].

Direct and indirect trauma to the lower limb may produce injury to the superior tibiofibular joint and cause the development of a fibular intraneural ganglion cyst [9]. In this case the history was negative for factors causing external nerve compression at the fibular head, such as habitual leg crossing.

Plain radiographs do not help in diagnosis of ganglion cyst but may be helpful to rule out bony lesions or outgrowths (a bony anomaly or fracture of the proximal part of fibula) [8] [9]. Also, they may be useful in excluding degenerative disc disease of the lumbar spine [8] [9].

An EMG study may be helpful to localize the level of the palsy and the extent of sensori-motor affection. Thus, electrophysiological studies are helpful in distinguishing whether it is a radiculopathy or peroneal nerve lesion. When more proximal lesions are suspected, a very useful strategy is to perform needle EMG examination of the lumbar paraspinal, gluteal and hamstring muscles [8] [9].

MRI is the noninvasive investigation of choice for diagnostic imaging and allows the lesion to be located and the distribution of the muscles supplying the peroneal nerve to be analyzed. Leon and Morano emphasize the advantages of MRI for assessing soft tissue tumors [25]. It may be difficult to differentiate a ganglion cyst from nerve sheath tumors and also solid masses on Magnetic resonance imaging. Ganglia characteristically present with low signal on T1-weighted images and high signal on T2-weighted images [26]. 
Ultrasonography (US) may be effective in showing the cystic nature of the mass (well-circumscribed) and in differentiating it from solid tumors (anechoic lesion) [8] [9] [27]-[29].

Currently, the gold standard treatment for a peroneal nerve palsy due to a peripheral nerve ganglion compression is surgical removal of the ganglion [2] [5] [8] [11] [15]-[17] [30] [31]. Careful surgical planning and proper delineation of the mass is most important. It is important to excise the articular connection of the cyst and its base in the superior tibiofibular joint so as to lessen the risk of recurrence. The prognosis of the peroneal nerve palsies of compressive origin is excellent. Instead recovery is much faster after operative decompression and will take place after few days or a few weeks [3] [15] [30].

We did a surgical decompression of the cyst and neurolysis of the peroneal nerve, combined with a intensive rehabilitation protocol which resulted in excellent recovery of nerve function. Because of the extensive branching pattern of the peroneal nerve in the area of the fibular head, we believe that needle aspiration carries a substantial risk for nerve damage. In addition, the high risk of recurrence of a ganglion following needle aspiration probably predicts less favourable outcomes.

Fabre et al. [12] reported 60 patients with peroneal nerve palsies, many idiopathic, who were treated with operative decompression. Postoperative recovery of motor function was good to excellent in $87 \%$ of those who had both sensory and motor involvement preoperatively. Decompression is recommended even for patients presenting with only sensory symptoms, if the symptoms have been substantiated by electrophysiological studies.

Local cyst recurrence postoperatively is reported and stresses the importance of articular branch ligation to avoid this complication [8] [23] [26] [32]. Simple excision of the ganglia is not sufficient. Other complications include traction injuries and perineural fibrosis with incomplete return of function [8]. Less commonly, nerve transection occurs, leading to permanent dysfunction [8].

Mehmet Erdil et al. [33] demonstrated a case report of a synovial cyst as the etiologic cause of sudden drop foot and stressed on the importance of considering synovial cyst around the knee in the differential diagnosis of deep peroneal neuropathy. They reported this case of an isolated DPN palsy secondary to a ganglion cyst.

Although the prevalence of these lesions is quite low (Ilahi et al. quoting a prevalence of $0.76 \%$ ) [34], it is important to consider this pathology as a differential diagnosis for patients with a gradually progressive presentation of peroneal nerve palsy causing foot drop in both adults and children.

\section{Highlights \& Teaching Points}

1) Ganglion cyst should be considered in the differential diagnosis of an acute or a subacute cause of foot drop depending on the symptomatology and clinical examination of the presenting patient. If back causes can be ruled out, stress should be on diagnostic clinical signs to detect a peripheral compressive lesion (Tinel's sign, swelling, etc.).

2) MRI and EMG/NCV form the mainstay investigations to diagnose/rule out the lesion and its localization.

3) A lumbar spine MRI should be avidly the first investigation to rule out a back pathology (especially in older patients).

4) Prompt diagnosis and operative decompression hold implications in the chances of recovery of the foot drop. The earlier the diagnosis and treatment, the better the prognosis.

5) The excision of the cyst and decompression of the nerve should be complete and meticulous. Reaching the base of the articular stalk of the cyst and the completeness of its removal are the governing factors for recurrence of the cyst.

\section{Conflicts of Interest}

None.

\section{Authors' Disclosure Statement}

The authors report no actual or potential conflict of interest in relation to this article.

\section{References}

[1] Strickland, J.W. and Steichen, J.B. (1977) Nerve Tumors of the Hand and Forearm. Journal of Hand Surgery, 2A, 285-291. http://dx.doi.org/10.1016/S0363-5023(77)80128-7 
[2] Ozden, R., Uruc, V., Kalaci, A. and Dogramaci, Y. (2013) Compression of Common Peroneal Nerve Caused by an Extraneural Ganglion Cyst Mimicking Intermittent Claudication. Journal of Brachial Plexus and Peripheral Nerve Injury, 8, 5 .

[3] Muramatsu, K., Hashimoto, T., Tominaga, Y., Tamura, K. and Taguchi, T. (2013) Unusual Peroneal Nerve Palsy Caused by Intraneural Ganglion Cyst: Pathological Mechanism and Appropriate Treatment. Acta Neurochirurgica, 155, 1757-1761. http://dx.doi.org/10.1007/s00701-013-1768-z

[4] Rawal, A., Ratnam, K.R., Yin, Q., Sinopidis, C. and Frostick, S.P. (2004) Compression Neuropathy of Common Peroneal Nerve Caused by an Extraneural Ganglion: A Report of Two Cases. Microsurgery, 24, 63-66. http://dx.doi.org/10.1002/micr.10203

[5] Tehli, O., Celikmez, R.C., Birgili, B., Solmaz, I. and Celik, E. (2011) Pure Peroneal Intraneural Ganglion Cyst Ascending along the Sciatic Nerve. Turkish Neurosurgery, 21, 254-258.

[6] Frank, D.A. and Sangimino, M.J. (2008) Peroneal Nerve Palsy Secondary to Ganglion Cyst of the Proximal Tibiofibular Joint in a Pediatric Patient. Healio Orthopaedics, 31, 86. http://dx.doi.org/10.3928/01477447-20080101-14

[7] Sultan, C. (1921) Ganglion der Nervenscheide des Nervus Peroneus. Zentralblatt für Chirurgie, 48, 963-965.

[8] Greer-Bayramoglu, R.J., Nimigan Bsc, A.S. and Gan, B.S. (2008) Compression Neuropathy of the Peroneal Nerve Secondary to a Ganglion Cyst. Canadian Journal of Plastic Surgery, 16, 181-183.

[9] Spinner, R.J., Desy, N.M. and Amrami, K.K. (2008) Sequential Tibial and Peroneal Intraneural Ganglia Arising from the Superior Tibiofibular Joint. Skeletal Radiology, 37, 79-84. http://dx.doi.org/10.1007/s00256-007-0400-9

[10] Hislop, H.J. and Montgomery, J. (1995) Daniels and Worthingham’s Muscle Testing: Techniques of Manual Examination. 6th Edition, WB Saunders, Philadelphia.

[11] Stamatis, E.D., Manidakis, N.E. and Patouras, P.P. (2010) Intraneural Ganglion of the Superficial Peroneal Nerve: A Case Report. Journal of Foot and Ankle Surgery, 49, 400.e1-400.e4. http://dx.doi.org/10.1053/j.jfas.2010.04.012

[12] Fabre, T., Piton, C., Andre, D., Lasseur, E. and Durandeau, A. (1998) Peroneal Nerve Entrapment. The Journal of Bone \& Joint Surgery, 80, 47-53.

[13] Mont, M.A., Dellon, A.L., Chen, F., Hungerford, M.W., Krackow, K.A. and Hungerford, D.S. (1996) The Operative Treatment of Peroneal Nerve Palsy. The Journal of Bone \& Joint Surgery, 78, 863-869.

[14] Mahaley, M.S. (1974) Ganglion of the Posterior Tibial Nerve: Case Report. Journal of Neurosurgery, 40, $120-124$. http://dx.doi.org/10.3171/jns.1974.40.1.0120

[15] Yazid Bajuri, M., Tan, B.C., Das, S., Hassan, S. and Subanesh, S. (2011) Compression Neuropathy of the Common Peroneal Nerve Secondary to a Ganglion Cyst. Clinica Terapeutica, 162, 549-552.

[16] Cebesoy, O., Tutar, E., Isik, M. and Arpacioglu, O. (2007) A Case of Isolated Giant Plexiform Neurofibroma Involving All Branches of the Common Peroneal Nerve. Archives of Orthopaedic and Trauma Surgery, 127, 709-712. http://dx.doi.org/10.1007/s00402-007-0303-1

[17] Waldschmidt, U. and Slongo, T. (2010) An Unusual Cause of Paralysis of the Peroneal Nerve-A Case Report. Journal of Pediatric Surgery, 45, 259-261. http://dx.doi.org/10.1016/j.jpedsurg.2009.09.039

[18] Ramelli, G.P., Nagy, L., Tuncdogan, E. and Mathis, J. (1999) Ganglion Cyst of the Peroneal Nerve: A Differential Diagnosis of Peroneal Nerve Entrapment Neuropathy. European Neurology, 41, 56-58. http://dx.doi.org/10.1159/000008002

[19] Ward, W.G. and Eckardt, J.J. (1994) Ganglion Cyst of the Proximal Tibiofibular Joint Causing Anterior Compartment Syndrome: A Case Report and Anatomical Study. The Journal of Bone \& Joint Surgery, 76, 1561-1564.

[20] Gibbon, A.J., Wardell, S.R. and Scott, R.D. (1999) Synovial Cyst of the Proximal Tibiofibular Joint with Peroneal Nerve Compression after Total Knee Arthroplasty. The Journal of Arthroplasty, 14, 766-768. http://dx.doi.org/10.1016/S0883-5403(99)90235-8

[21] Edwards, M.S., Hirigoyen, M. and Burge, P.D. (1995) Compression of the Common Peroneal Nerve by a Cyst of the Lateral Meniscus: A Case Report. Clinical Orthopaedics and Related Research, 316, 131-133.

[22] Huaux, J.P., Malghem, J., Maldague, B., Noel, H., Rombouts, J.J., Courtois, C. and Nagant de Deuxchaisnes, C. (1986) Pathology of the Upper Peroneotibial Joint. History of Cysts. Apropos of 4 Cases. Revue du Rhumatisme et des Maladies Ostéo-Articulaires, 53, 723-726.

[23] Rein, S., Weindel, S., Schaller, H.-E., Mittelbronn, M. and Schmidt, G. (2005) Peroneal Nerve Palsy Caused by a Recurrent Proximal Tibiofibular Joint Ganglion-A Case Report and Review of the Literature. Handchirurgie Mikrochirurgie Plastische Chirurgie, 37, 267-275. http://dx.doi.org/10.1055/s-2005-865896

[24] Anselmi, S.J. (2006) Common Peroneal Nerve Compression. Journal of the American Podiatric Medical Association, 96, 413-417. http://dx.doi.org/10.7547/0960413 
[25] Leon, J. and Marano, G. (1987) MRI of Peroneal Nerve Entrapment Due to Ganglion Cyst. Magnetic Resonance Imaging, 5, 307-309. http://dx.doi.org/10.1016/0730-725X(87)90009-9

[26] Iverson, D.J. (2005) MRI Detection of Cysts of the Knee Causing Common Peroneal Neuropathy. Neurology, 65, 1829-1831. http://dx.doi.org/10.1212/01.wnl.0000187098.42938.b6

[27] Lucchetta, M., Liotta, G.A., Briani, C., Fernandez Marquez, E.M., Martinoli, C., Coraci, D. and Padua, L. (2011) Ultrasound Diagnosis of Peroneal Nerve Variant in a Child with Compressive Mononeuropathy. Journal of Pediatric Surgery, 46, 405-407. http://dx.doi.org/10.1016/j.jpedsurg.2010.09.055

[28] Visser, L.H. (2006) High-Resolution Sonography of the Common Peroneal Nerve: Detection of Intraneural Ganglia. Neurology, 67, 1473-1475. http://dx.doi.org/10.1212/01.wnl.0000240070.98910.bc

[29] Bayrak, A.O., Bayrak, I.K., Battaloglu, E., Ozes, B., Yildiz, O. and Onar, M.K. (2014) Ultrasonographic Findings in Hereditary Neuropathy with Liability to Pressure Palsies. Neurological Research, 37, 106-111. http://dx.doi.org/10.1179/1743132814Y.0000000411

[30] de Schrijver, F., Simon, J.P., de Smet, L. and Fabry, G. (1998) Ganglia of the Superior Tibiofibular Joint: Report of Three Cases and Review of the Literature. Acta Orthopaedica Belgica, 64, 233-241.

[31] Yang, L.J.S., Gala, V.C. and McGillicuddy, J.E. (2006) Superficial Peroneal Nerve Syndrome: An Unusual Nerve Entrapment-Case Report. Journal of Neurosurgery, 104, 820-823. http://dx.doi.org/10.3171/jns.2006.104.5.820

[32] Dubuisson, A.S. and Stevenaert, A. (1996) Recurrent Ganglion Cyst of the Peroneal Nerve: Radiological and Operative Observations: Case Report. Journal of Neurosurgery, 84, 280-283. http://dx.doi.org/10.3171/jns.1996.84.2.0280

[33] Erdil, M., Ozkanb, K., Ozkanc, F.U., Bilsel, K., Turkmenb, I., Senold, S. and Sarar, S. (2013) A Rare Cause of Deep Peroneal Nerve Palsy Due to Compression of Synovial Cyst-Case Report. International Journal of Surgery Case Reports, 4, 515-517. http://dx.doi.org/10.1016/j.ijscr.2012.11.028

[34] Ilahi, O.A., Younas, S.A., Labbe, M.R. and Edson, S.B. (2003) Prevalence of Ganglion Cysts Originating from the Proximal Tibiofibular Joint: A Magnetic Resonance Imaging Study. Arthroscopy, 19, 150-153. http://dx.doi.org/10.1053/jars.2003.50007

\section{Abbreviations}

MRI: Magnetic Resonance Imaging.

DPN: Deep Peroneal Nerve.

CPN: Common Peroneal Nerve.

SPN: Superficial Peroneal Nerve.

EMG/NCV: Electromyography/Nerve Conduction Studies. 